\title{
The Regeneration of Brachial Nerves of Contralateral Origin into Denervated Fused Newt Forelimbs ${ }^{1,2,3}$
}

\author{
HARRY G. GOSHGARIAN 4 \\ Department of Anatomy, University of Michigan, \\ Ann Arbor, Michigan 48104
}

\begin{abstract}
Previous studies involving the end-to-end fusion of the forelimbs of the adult newt have demonstrated that new limbs can regenerate from the transected ends of proximo-distally reversed limb segments. The limb regeneration could only have been initiated by nerve fibers of contralateral origin. The purpose of the present study is to describe histologically the manner in which nerve fibers of contralateral origin regenerate through the junction of fused limbs into the opposite limb. The first sign of nerve regeneration into the opposite limb was observed at eight days post fusion. The nerves crossed over into the opposite, originally denervated limb in a highly dispersed manner. These nerve fibers eventually aggregated, however, either under the skin or within persisting nerve trunks. By 19 days post fusion the nerve fibers had reached the elbow region of the originally denervated limb and by 25 days they were seen at the most proximal extent of the limb. The diameters of the axons seemed smaller than the diameters of regenerating axons observed in non-fused newt forelimbs.
\end{abstract}

The role of nerves in the regeneration of amphibian extremities has been well documented (reviewed by Rose, '48; Singer, '52, '59; Thorton, '68). In the adult newt, as in many other amphibians, a denervated forelimb will not regenerate after amputation. A specific quantity of intact nerve fibers must be present in the limb before regeneration can occur (Singer, '46, '47). Furthermore, if the number of nerve fibers is significantly less than the number normally present in the limb, the regenerate is morphologically complete, but reduced in length and volume (Singer and Egloff, '49).

Carlson et al. ('74) amputated both forelimbs of the adult newt through the distal radius and ulna and then surgically fused the limbs end-to-end. In one experimental series the right forelimb was denervated at the time of fusion and it was subsequently transected through the humerus. After these surgical procedures the right limb was attached to the newt only at the site of fusion with the left limb. An abnormally small, yet morphologically complete forelimb, regenerated from the site of transection of the right limb in all of the long term animals. Innervation at the wound site sufficient to produce limb regeneration could only have been provided by branchial nerves of contralateral origin which had regenerated into the previously denervated limb. Thus, the regenerating nerve fibers were presented with an abnormally elongated extremity, a portion of which was reversed in its proximodistal polarity.

The present investigation was carried out to provide information about: (1) the manner in which regenerating nerve fibers crossed the zone of fusion of the two limbs, and (2) the time course of progression of regenerating nerve fibers towards the proximal regions of the contralateral limb. Other experiments dealing with functional activities of the nerve fibers in the new territory will be the subject of a subsequent paper.

\section{MATERIALS AND METHODS}

Forty six adult male and female newts, Notophthalmus viridescens, were kept in groups of five at $21^{\circ} \pm 2^{\circ}$ and fed twice a week with beef liver. Before all opera-

\footnotetext{
1 Supported by NIH Training Grant 5 T01 GM 00312 15 and a grant from the Muscular Dystrophy Association of America to Dr. Bruce M. Carlson.

2 Reported earlier in abstract form (Goshgarian, '75a).

3 This material is part of a thesis for the degree of Doctor of Philosophy, University of Michigan.

4 Present address: Bldg. 9, Rm. 1E125, National Institutes of Health, 9000 Rockville Pike, Bethesda, Maryland 20014.
} 
tions newts were anesthetized by immersion into a 1:1000 tap water solution of MS-222 (tricaine methane sulfonate, Sandoz) or ethyl m-aminobenzoate methanesulfonate (Eastman).

The left forelimb of all animals was denervated by severing spinal nerves three through five. To prevent nerve regeneration the brachial plexus of each left forelimb was retransected every ten days post fusion and six days prior to sacrifice. A $2 \mathrm{~mm}$ segment of the humerus was removed from the right non-denervated forelimb to prevent the animals from pulling their arms apart.

All forelimbs were surgically fused after denervation and humerectomy. Each limb was amputated through the distal radius and ulna and any protruding bones were trimmed. The dorsoventral and anteroposterior axes of the amputated limbs were aligned, and the limbs were joined end-to-end by three $7-0$ sutures. Animals were placed in a coldroom $\left(4-8^{\circ}\right)$ for seven days following the fusion operation to reduce their activity during the early healing period.

All animals were sacrificed between one and 31 days post fusion. The fused limbs of the animals were embedded in paraffin and longitudinal serial sections were prepared for silver staining by a modification of Palmgren's ('60) silver impregnation technique. A calibrated ocular micrometer disk was used to measure the progress of nerve regeneration.

\section{RESULTS}

The severed nerves found in the denervated limb were in an advanced stage of degeneration by five days post fusion. As early as three to five days post fusion a few regenerating nerve fibers from the contralateral non-denervated limb were seen among the cells of a well-developed wound epithelium at the junction of the fused limbs. By six to 12 days the fusion zone epithelium was invaded by numerous regenerating nerve fibers. Axons increased in number by branching and sprouted from the amputated ends of the nerve trunks of the non-denervated limb. The nerve fibers appeared to be without neurilemmal or endoneurial components and were dispersed irregularly among the epithelial cells.
The first intact nerve fibers were seen in the originally denervated limb on the eighth day post fusion and were sparsely distributed beneath the skin near the junction of the limbs. Axons increased in the distal region of the originally denervated limb by the end of the second week. The majority of axons were not distinctly fasciculated. Rather, small areas within the limb contained three or four loosely arranged nerve fibers (figs. 1, 2).

By 18-20 days post fusion regenerating axons from the non-denervated limb could be clearly seen within the originally denervated limb. The axons were highly dispersed at the distal end of the originally denervated limb; but became fasciculated after regenerating about $1 \mathrm{~mm}$ into the limb. Fasciculation occurred either under the skin (fig. 3) or within nerve trunks which had persisted after the degeneration of the normal nerve component of the denervated limb (fig. 4). The persisting nerve trunks contained not only the regenerating axons of contralateral origin, but also the axonal remnants of the degenerated normal nerve component of the limb.

An assessment of the degree of axonal dispersal at the fusion zone was obtained after the study of numerous serial sections cut through the junction of the fused limbs. In one animal, for example 34 serial sections were examined before regenerating axons in the persisting nerve trunks were traced back to the intact nerves of the non-denervated limb. Since the serial sections were cut at $15 \mu$ each, an approximate distance of $510 \mu$ was involved in the dispersal of axons through the fusion zone. A diagramatic composition of the 34 serial sections representing the $510 \mu$ distance shows the manner in which nerve fibers become dispersed at the fusion zone and become reaggragated into distinct bundles after regenerating into the originally denervated limb (fig. 5).

In the four limbs fixed at 19 days post fusion, regenerating fibers of contralateral origin reached the elbow region of the originally denervated limb. In three of these limbs, nerve fibers were traced between 420 and $2,800 \mu$ from the elbow into the upper arm. The fibers of the fourth limb had not regenerated as far as the elbow and were seen $200 \mu$ away. In three out of four animals whose limbs 
had been fused for 22 days and in every animal with limbs fused for 25 days or longer, nerve fibers had reached the proximal end of the originally denervated limb at the level of the head of the humerus. In several animals the axons appeared thinner than regenerating axons of the same age in non-fused newt forelimbs. In order to reach the head of the humerus of the originally denervated limb, the nerves had to elongate to almost twice their normal length.

\section{DISCUSSION}

Electrophysiological and behavioral studies reported elsewhere have shown that brachial nerves of contralateral origin not only regenerate into the originally denervated limb, but are also capable of establishing functional connections with the tissues of this limb (Goshgarian, '75b). Sensory innervation appeared long before signs of motor innervation were observed.

There have been several reports in which the proximo-distal axis of urodele limbs was surgically reversed in studies on limb regeneration (Butler, '51; Deck, '55; Dent, '54). In all of these investigations the limb was amputated distally, implanted into the lateral body wall and then reamputated through the shoulder. Evidence that the limbs became innervated by foreign nerves existed since many displayed signs of regeneration at the transection site through the shoulder. It should be stressed, however, that the limb regeneration was induced by nerves originating from the same side of the animal as the transplanted limb. Furthermore, these ipsilateral nerves regenerated a shorter distance than the normal length of the limb to initiate the limb regeneration.

The present study shows that brachial nerves originating on the contralateral side of the animal are capable of elongation to almost twice their normal length. The histological results have demonstrated that the nerves of contralateral origin are fasciculated either under the skin or within nerve trunks which had persisted after the degeneration of the originally denervated limb's normal nerve component. Similar results were obtained by Deck ('55) after he reversed the polarity of larval urodele limbs and studied their innervation. The fasciculation of the contralateral nerves within the originally denervated limb is significant since the nerves could be easily dissected from the limb in regeneration studies involving heterotopic nerve transplantation. The elongated nerves could be transplanted to brachial and nonbrachial sites to augment the normal innervation of these regions. The results of long term studies on the interaction of nerves of opposite regenerating polarity in fused newt forelimbs (Goshgarian, '75b) have indicated that the dispersal of nerves through the fusion zone is gradually decreased during the second and third months post fusion. Thus, a complete dissection of the nerves from the fused limbs should be possible.

Supernumerary limb regenerates appeared at the junction of limbs fused without denervation indicating that nerves will not grow into territories already innervated by intact nerves (Carlson et al., 74). Aguilar et al. (73) postulated that the mechanism for the regulation of peripheral nerve fields in salamander extremities is the axoplasmic transport of a trophic factor which inhibits axonal sprouting from adjacent nerves when an area within the limb is innervated. Alternatively, Cass et al. (73) have shown that nerves normally innervating specific areas within a limb can sprout and innervate new areas within the same limb provided that these new areas are denervated. The present investigation shows that when one limb is denervated, contralateral nerve fibers cross through the fusion zone and enter into the denervated limb. Thus, reversal of the normal proximo-distal limb gradient does not inhibit the ingrowth of nerves regardless of their origin. The only factor which inhibits the ingrowth of nerves is the presence of a normal innervation.

During the first eight days after fusion the total distance traversed by regenerating fibers was less than $1 \mathrm{~mm}$. In the next two weeks, however, the fibers grew an additional $10 \mathrm{~mm}$. The obvious differences in rate of nerve regeneration are probably due to an initial delay before axonal elongation as well as obstruction by tissue debris at the junction of the fused limbs. Weiss ("45) noted that physical boundaries influence the rate of nerve regeneration, and observed that differences in the rate need not be due to any 
intrinsic property of the neuron itself. Once the nerves passed through the fusion zone, however, individual variations in the rate of nerve regeneration were noted at 19 and 22 days post fusion.

By 25 days the innervation of the contralateral limb was complete in all animals since fibers were always seen at the level of the limb containing the head of the humerus. No attempt was made to measure the extent of nerve regeneration beyond the limb into the lateral body wall since identification of the axons would be difficult because of the masking effect of sprouted axons from spinal nerves two and six (Singer, '46). The ability of the nerves to regenerate to even greater distances than the end of the originally denervated limb has been demonstrated, however, in an earlier work from our laboratory (Carlson et al., '74). Cross sections through the regenerate emanating from the end of the originally denervated transected limb contained nerve fibers. In order to reach these levels the brachial nerves of contralateral origin had to regenerate to over 2.5 times their normal length.

The diameter of the axons in several limbs appeared smaller at the level of the head of the humerus than the diameter of regenerating axons of the same age in non-fused newt forelimbs. The obvious reduction in the amount of axoplasm in the smaller axons may be the reason for the reduced length and volume of the regenerates which appeared at the transection plane in the investigation of Carlson et al. (74). A detailed quantitative study on both the number and size of the axons at this level of the originally denervated limb must be carried out, however, before this conclusion can be verified.

\section{ACKNOWLEDGMENTS}

The author wishes to express his appreciation to Drs. Thomas G. Connelly and Bruce $M$. Carlson not only for their critical evaluation of this manuscript, but also for the years of helpful suggestions and guidance. Art work was done by $\mathrm{Mr}$. William Brudon.

\section{LITERATURE CITED}

Aguilar, C. E., M. A. Bisby, E. Cooper and J. Diamond 1973 Evidence that axoplasmic transport of trophic factors is involved in the regulation of peripheral nerve fields in salamanders. J. Physiol., 234: 449-464.

Butler, E. G. 1951 The mechanics of blastema formation and regeneration in urodele limbs of reversed polarity. Trans. N. Y. Acad. Sci., 13: 164-167.

Carlson, B. M., S. E. Civiletto and H. G. Goshgarian 1974 Nerve interactions and regenerative processes occurring in newt limbs fused end to end. Dev. Biol., 37: 248-262.

Cass, D. T., T. J. Sutton and R. F. Mark 1973 Competition between nerves for functional connexions with axolotl muscles. Nature, 243: 201 203.

Deck, J. D. 1955 The innervation of urodele limbs of reversed proximo-distal polarity. J. Morph., 96: 301-331.

Dent, J. N. 1954 A study of regenerates emanating from limb transplants with reversed proximodistal polarity in the adult newt. Anat. Rec., 118: 841-856.

Goshgarian, H. G. 1975a Peripheral nerve regeneration occurring in surgically fused newt forelimbs. Anat. Rec., 181: 364 .

$1975 \mathrm{~b}$ Interaction of Nerves of Opposite Regenerating Polarity in Fused Newt Forelimbs. Ph.D. dissertation, University of Michigan.

Palmgren, A. 1960 Specific silver staining of nerve fibers. I. Technique for vertebrates. Acta Zool., 41 : 239-265.

Rose, S. M. 1948 The role of nerves in amphibian limb regeneration. Ann. N. Y. Acad. Sci., 49: 818-833.

Singer, M. 1946 The nervous system and regeneration of the forelimb of aduit triturus. V. The influence of number of nerve fibers, including a quantitative study of limb innervation. J. Exp. Zool., 101 : 299-337.

1947 The nervous system and regeneration of the forelimb of adult triturus. VI. A further study of the importance of nerve number, including quantitative measurements of limb innervation. J. Exp. Zool., 104: 223-250.

1952 The influence of the nerve in regeneration of the amphibian extremity. Quart. Rev. Biol., 27 : 169-195.

1959 The influence of nerves on regeneration. In: Regeneration in Vertebrates. C. S. Thornton, ed. Univ. Chicago Press, Chicago, pp. 59-80.

Singer, M., and F. R. L. Egloff 1949 The nervous system and regeneration of the forelimb of adult triturus. VIII. The effect of limited nerve quantities on regeneration. J. Exp. Zool., 111 . 295-314.

Thornton, C. S. 1968 Amphibian limb regeneration. Adv. Morphogen., 7: 205-249.

Weiss, P. 1945 Experiments on cell and axon orientation in vitro: the role of colloidal exudates in tissue organization. J. Exp. Zool., 100: 353-386. 
PLATES 


\section{PLATE 1}

\section{EXPLANATION OF FIGURES}

1 Newt forelimbs 17 days post fusion: low power view for orientation. Arrows point to fusion junction. The originally denervated limb is located above the arrows; the non-denervated limb is located below the arrows. The area within the upper black rectangle is enlarged in figure 4 . The area within the lower white rectangle is enlarged in figure 2. Modified Palmgren stain. $\times 12.5$.

2 Loosely arranged nerve fibers within the originally denervated limb (arrows at A). Seventeen days post fusion. Modified Palmgren stain. $\times 100$. 


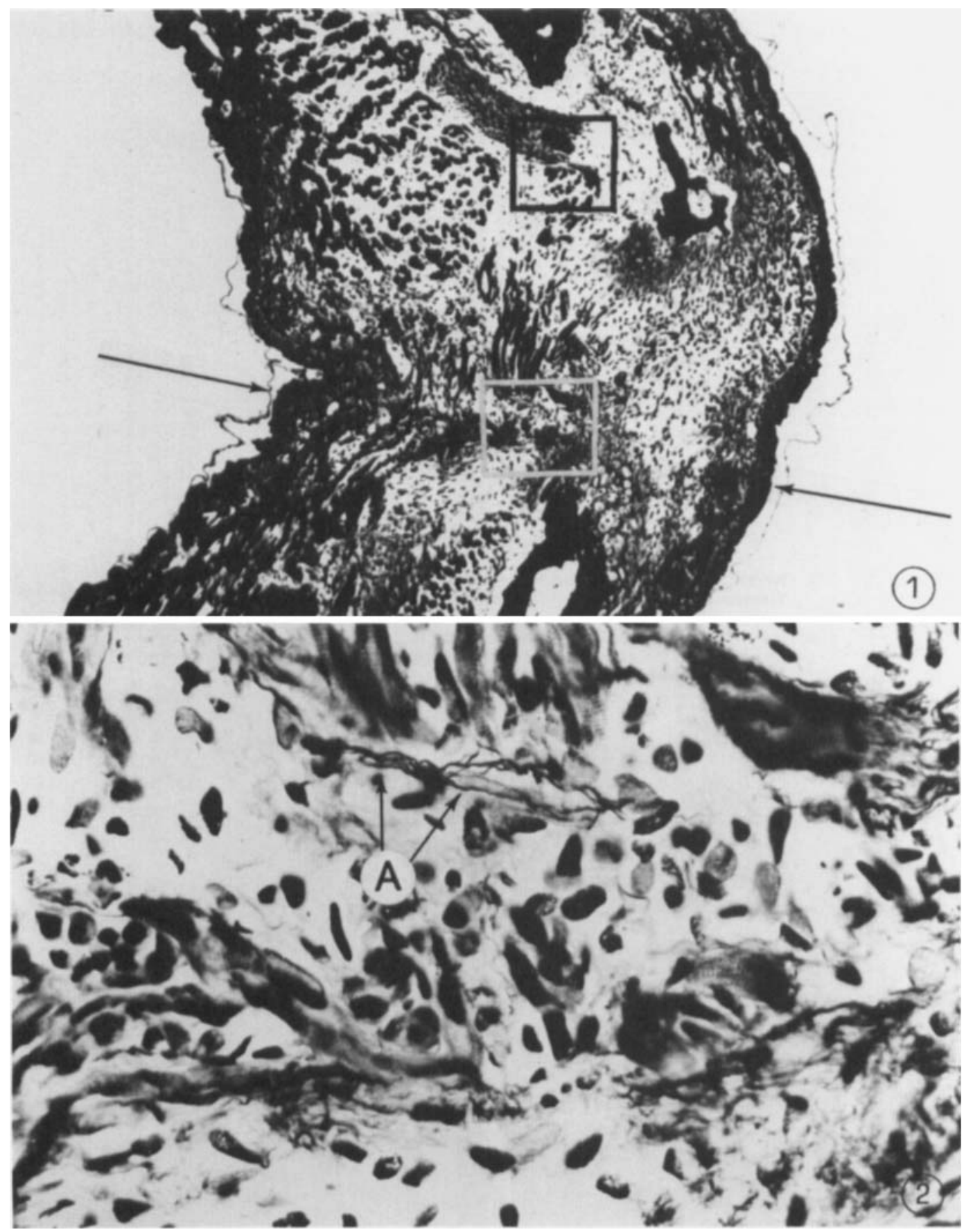


PLATE 2

EXPLANATION OF FIGURES

3 Nerve fibers (arrows) under the skin of the originally denervated limb. Sixteen days post fusion. Modified Palmgren stain. $\times 100$.

4 Fascicle of nerves of contralateral origin (N) entering persisting nerve trunk (P) of originally denervated limb. Seventeen days post fusion. Modified Palmgren stain. $\times 100$. 


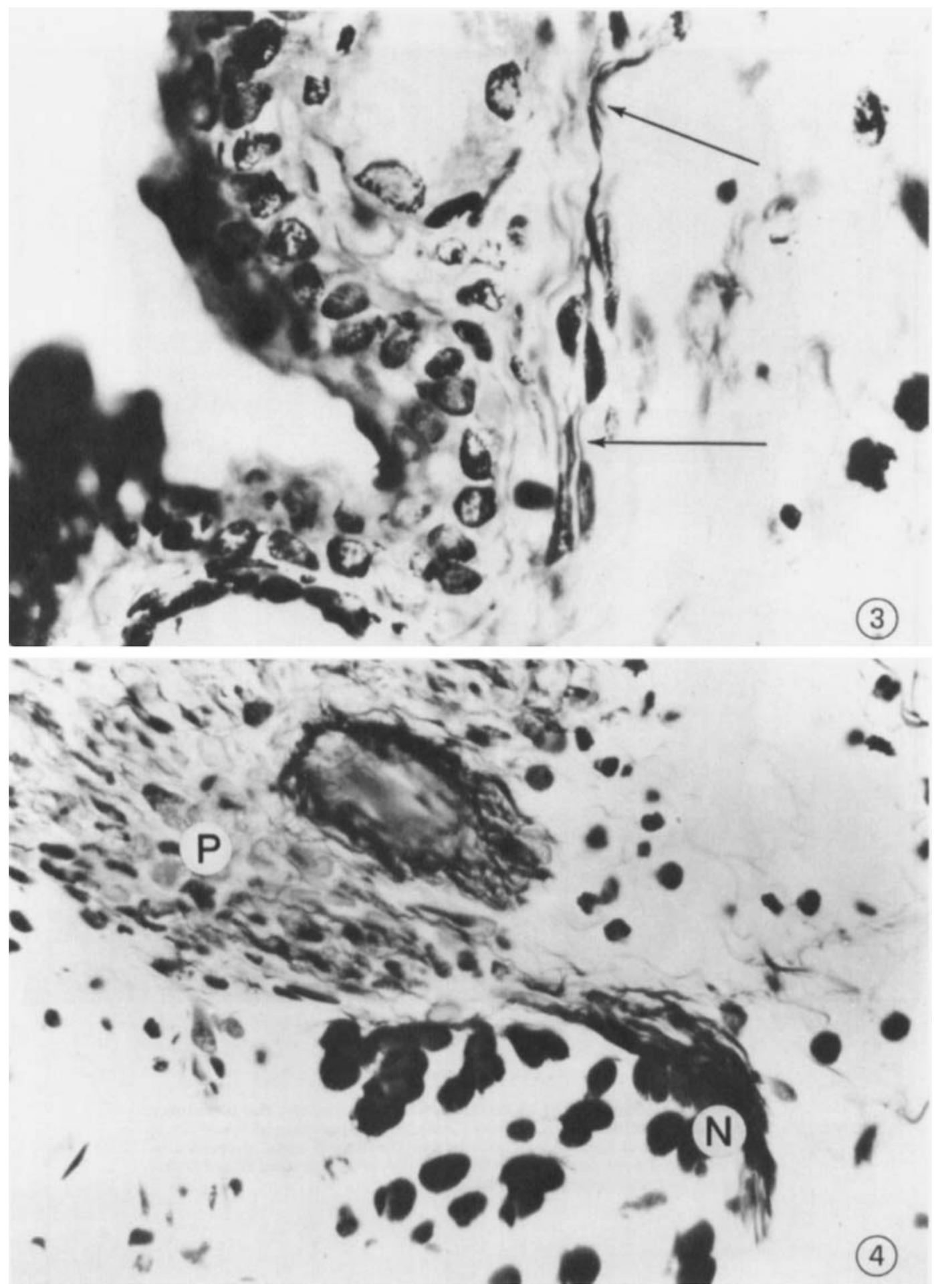




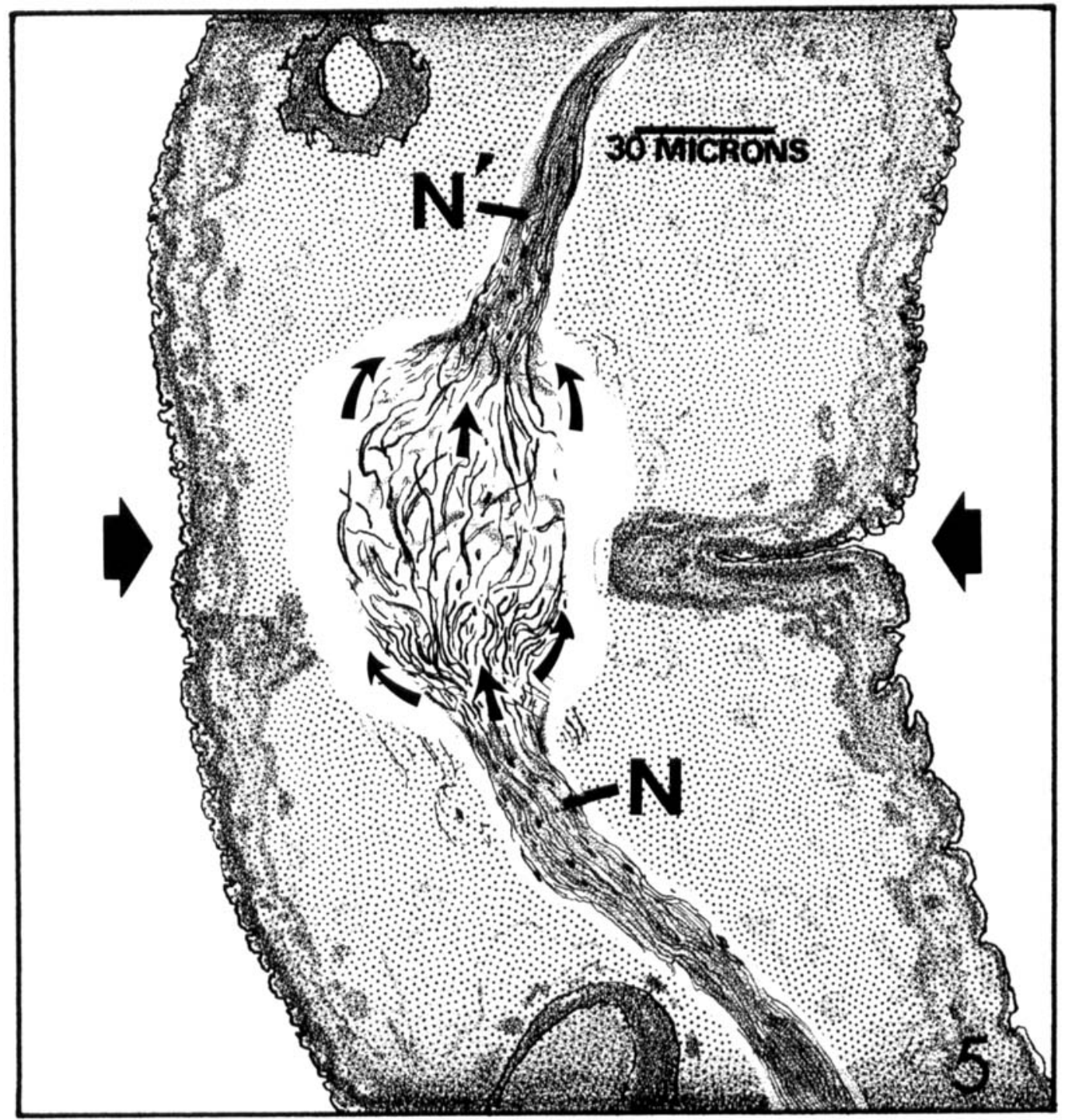

EXPLANATION OF FIGURE

5 Diagramatic composite of 34 serial sections cut through the junction of fused newt forelimbs. Large arrows indicate the junction of fused limbs. Although axonal spouting occurs before the fusion zone, dispersal occurs as axons pass through the fusion zone. $\mathrm{N}^{\prime}$, persisting nerve trunk; $\mathrm{N}$, int act nerve of non-denervated limb. 